\title{
Corrigendum: Regional and Temporal Differences in Brain Activity With Morally Good or Bad Judgments in Men: A Magnetoencephalography Study
}

\author{
Hirotoshi Hiraishi 1,2, Takashi Ikeda ${ }^{2,3}$, Daisuke N. Saito ${ }^{2,4}$, Chiaki Hasegawa ${ }^{2}$, \\ Sachiko Kitagawa ${ }^{2}$, Tetsuya Takahashi ${ }^{2}$, Mitsuru Kikuchi ${ }^{2,3,5 *}$ and Yasuomi Ouchi ${ }^{1 *}$ \\ ${ }^{1}$ Department of Biofunctional Imaging, Preeminent Medical Photonics Education and Research Center, Hamamatsu \\ University School of Medicine, Hamamatsu, Japan, ${ }^{2}$ Research Center for Child Mental Development, Kanazawa University, \\ Kanazawa, Japan, ${ }^{3}$ United Graduate School of Child Development, Osaka University, Kanazawa University, Hamamatsu \\ University School of Medicine, Chiba University and University of Fukui, Osaka, Japan, ${ }^{4}$ Department of Psychology, Yasuda \\ Women's University, Hiroshima, Japan, ${ }^{5}$ Department of Psychiatry and Behavioral Science, Kanazawa University, Kanazawa, \\ Japan
}

Keywords: moral judgment, MEG, brain activity, connectivity, morally good judgment, morally bad judgment

\section{A Corrigendum on}

\section{OPEN ACCESS}

Edited and reviewed by:

Paul E. M. Phillips,

University of Washington,

United States

*Correspondence:

Mitsuru Kikuch

mitsuruk@med.kanazawa-u.ac.jp Yasuomi Ouchi

Ouchi@hama-med.ac.jp

Specialty section:

This article was submitted to Decision Neuroscience,

a section of the journal

Frontiers in Neuroscience

Received: 04 August 2021 Accepted: 23 September 2021 Published: 21 October 2021

Citation:

Hiraishi H, Ikeda T, Saito DN, Hasegawa C, Kitagawa S, Takahashi T, Kikuchi M and Ouchi Y (2021) Corrigendum: Regional and Temporal Differences in Brain Activity With Morally Good or Bad Judgments in Men: A Magnetoencephalography Study. Front. Neurosci. 15:753147. doi: 10.3389/fnins.2021.753147
Regional and Temporal Differences in Brain Activity With Morally Good or Bad Judgments in Men: A Magnetoencephalography Study

by Hiraishi, H., Ikeda, T., Saito, D. N., Hasegawa, C., Kitagawa, S., Takahashi, T., Kikuchi, M., and Ouchi, Y. (2021). Front. Neurosci. 15:596711. doi: 10.3389/fnins.2021.596711

In the original article, there was a mismatch between the figures and their legends as published. The captions for Figures 1 and 2 were switched, and the captions for Figures 3 and 4 had also switched in the published article. As a result, all the figures and their figure legends were mismatched. To resolve this, the image currently used for Figure 2 should instead be Figure 1, and the image currently used for Figure 1 should instead be Figure 2. Similarly, for Figures 3 and 4, the images should be swapped, so that the image currently labeled as Figure 3 becomes Figure 4, and the image labeled as Figure 4 becomes Figure 3. The captions are then correct as they are. The correct figures appear below.

Additionally, in the published article, there was an error in affiliation 3 as published. Instead of "United Graduate School of Child Development, Osaka University, Kanazawa University, Hamamatsu University School of Medicine, Chiba University and University of Fukui, Fukui, Japan", it should be "United Graduate School of Child Development, Osaka University, Kanazawa University, Hamamatsu University School of Medicine, Chiba University and University of Fukui, Osaka, Japan”.

Lastly, in the original article, there was a duplication of a description provided in the Materials and Methods section. The following sentence appearing at the end of the this section has been removed:

"The same number of good and bad situations were presented in Phase 3 (96 situations each), and the number of neutral situations was 48 . They were presented in a random order." The corrected paragraph is shown below.

The participants completed a set of tasks (Figure 1) that were modified from the previous study by Decety and Cacioppo (2012). During the task, the participants watched a series of three-frame video clips that were presented centrally on a monitor screen. Before a story began, a fixation cross appeared for 1,000 msec. Following the fixation cross, the first frame and the second frame from the 
video clip, which were each $500 \mathrm{msec}$ long, were displayed to establish the scene; the third frame (Phase 3) was 1,000 msec long and displayed a scene requiring a moral judgment. After Phase 3 disappeared, the question "Do you think this was good or bad?" in Japanese was displayed for 1,000 msec. The participants were asked to judge by pressing a button with a right thumb if a behavior of a person in pictures was considered to be morally good or pressing a button with a left thumb if morally bad during

\section{REFERENCES}

Decety, J., and Cacioppo, S. (2012). The speed of morality: a highdensity electrical neuroimaging study. J. Neurophysiol. 108, 3068-3072. doi: 10.1152/jn.00473.2012

Publisher's Note: All claims expressed in this article are solely those of the authors and do not necessarily represent those of their affiliated organizations, or those of the publisher, the editors and the reviewers. Any product that may be evaluated in a period of $1,000 \mathrm{msec}$. If they judged it as a morally neutral behavior, they did not push any button. The same number of good and bad situations were presented in Phase 3 (96 situations each), and the number of neutral situations was 48 . They were presented in a random order.

The authors apologize for these errors and state that they do not change the scientific conclusions of the article in any way. The original article has been updated.

this article, or claim that may be made by its manufacturer, is not guaranteed or endorsed by the publisher.

Copyright (C) 2021 Hiraishi, Ikeda, Saito, Hasegawa, Kitagawa, Takahashi, Kikuchi and Ouchi. This is an open-access article distributed under the terms of the Creative Commons Attribution License (CC BY). The use, distribution or reproduction in other forums is permitted, provided the original author(s) and the copyright owner(s) are credited and that the original publication in this journal is cited, in accordance with accepted academic practice. No use, distribution or reproduction is permitted which does not comply with these terms. 


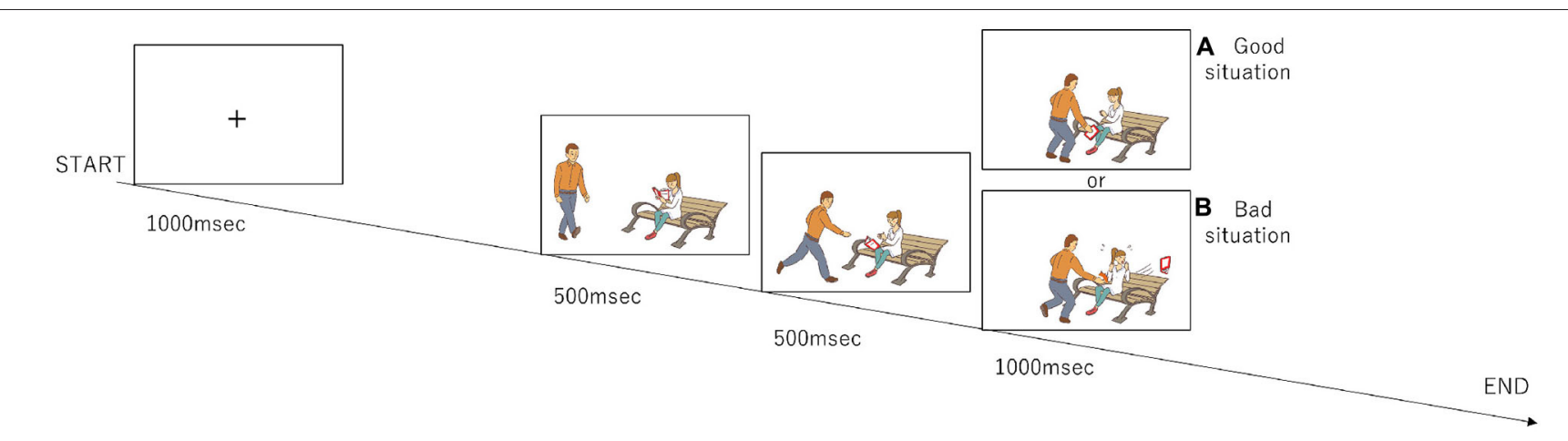

FIGURE 1 | Design of the moral judgment task. The participants were presented three-frame video clips about morally positive, negative, and neutral contexts and were asked to judge morality as soon as possible after the presentation of the third picture. The numbers of presentations for the positive, negative, and neutral stories are 96,96 , and 48 , respectively. 


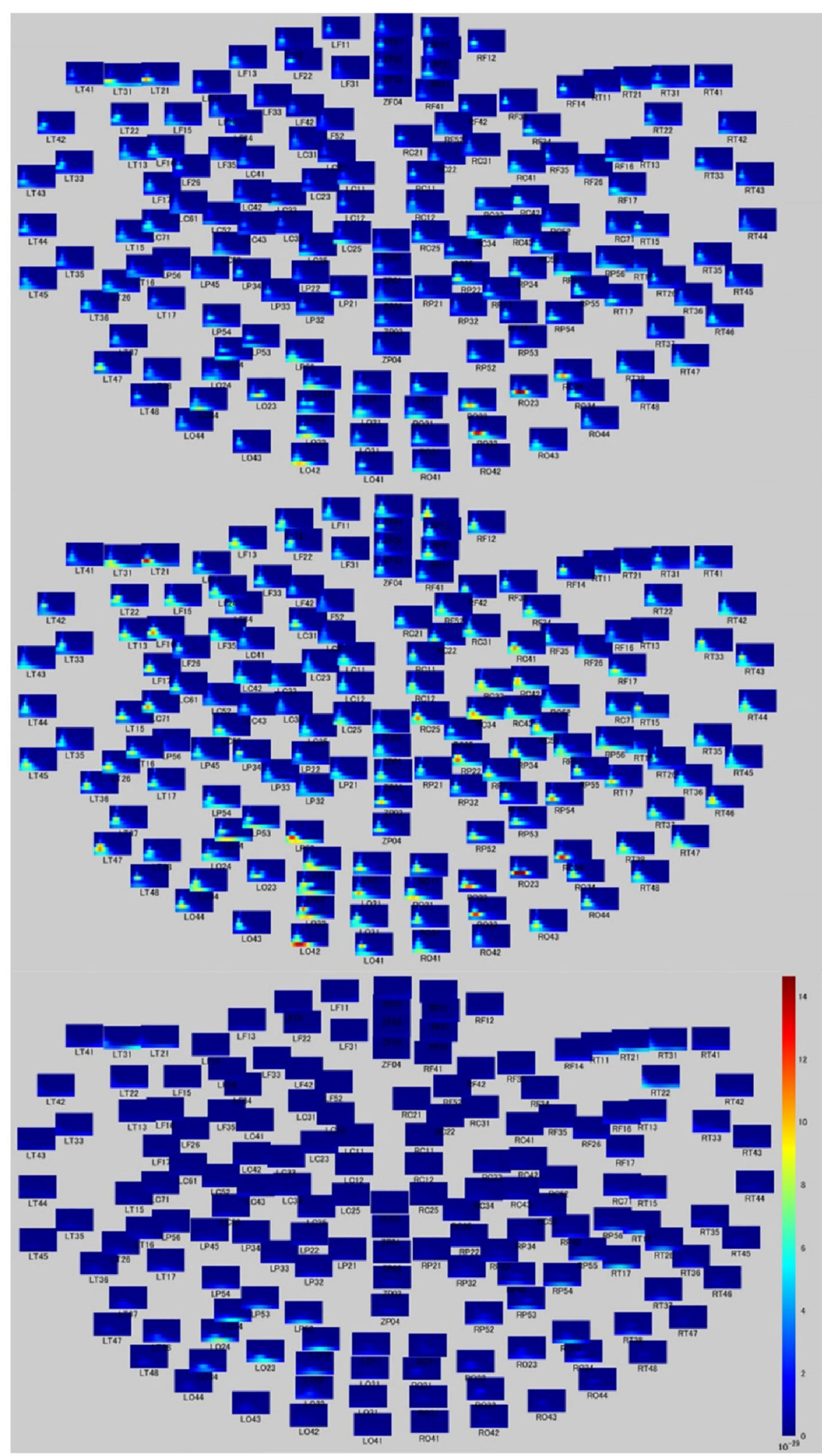

FIGURE 2 | Time-frequency relationship map during moral judgment. Time-frequency figures on whole head during MGJ (upper), MBJ (middle), and MNJ (lower) conditions. The $X$-axis of each small panel indicates the time from $200 \mathrm{~ms}$ before to 1,000 $\mathrm{ms}$ after a phase three picture presentation, and the $Y$-axis indicates the $\mathrm{Hz}$ from 0 to 120. The color bar denotes the power (signal units $2 / \mathrm{Hz} \times 10-29$ ) from 0 to 15. (A) The third frame that represents a morally good situation. (B) The third frame that represents a morally bad situation. 

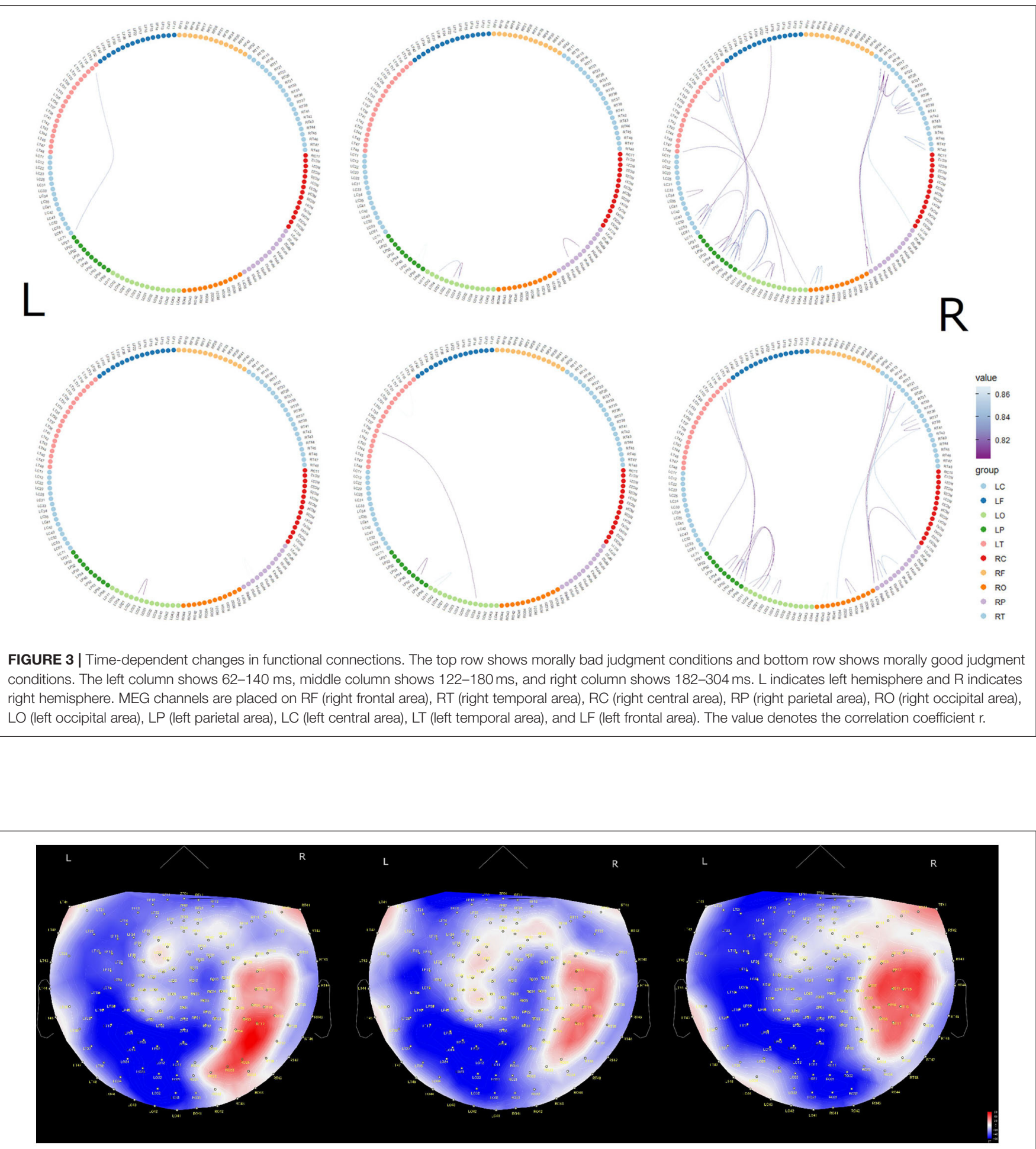

FIGURE 4 | Time-dependent changes in activated brain areas on 2D cap images. Time windows: left shows $62-140 \mathrm{~ms}$, middle shows $122-180 \mathrm{~ms}$ and right shows 182-304 ms. L, left hemisphere; R, right hemisphere. The color bar denotes amplitude (fT) from -60 (blue) to 60 (red). 